\title{
Manejo de la fractura-luxación Interfalángica proximal mediante fijador externo tipo Suzuki. Protocolo de tratamiento y resultados preliminares
}

\section{Proximal Interphalangeal Fracture-Dislocation Management by External Fixator Type Suzuki. Treatment Protocol and Preliminary Outcomes}

\author{
Rafael Laredo Rivero ${ }^{1,2}$ Bárbara Maria Pérez Fabra ${ }^{1}$ \\ Roberto Cibantos Martínez ${ }^{1}$ David Gomez Garrido ${ }^{3}$

\footnotetext{
${ }^{1}$ Servicio de Cirugía Ortopédica y Traumatología, Complejo Hospitalario de Toledo, Toledo, Spain

2 Unidad de Cirugía de Mano, Hospital Quiron Toledo, Spain

${ }^{3}$ Servicio de Cirugía Ortopédica y Traumatología, Hospital General de Villalba, Madrid, Spain
}

Rev Iberam Cir Mano 2019;47:77-83.
Victor Delgado Alcalá ${ }^{1}$

Javier Cervera Irima ${ }^{3}$

\section{Resumen \\ Palabras clave \\ - articulación interfalángica proximal \\ - fractura - luxación \\ - fijador externo dinámico}

Abstract
Introducción La fractura-luxación de la articulación interfalángica proximal de la mano es frecuente en la práctica clínica, sin embargo, no existe un consenso ni indicaciones definidas para cada tipo de fractura, lo que supone un importante campo de confusión y su tratamiento continúa siendo hoy en día un reto no resuelto. Nos preguntamos si es posible simplificar este algoritmo y utilizar el fijador tipo Suzuki de forma protocolizada para manejar la gran mayoría de lesiones con indicación quirúrgica.

Métodos Se recogieron 15 pacientes con patrones de fractura de tipo 1 (66,6\%), tipo 2 (6,6\%), tipo $3(20 \%)$ y tipo 5 (6,6\%) según la clasificación de Seno entre 2011 y 2019 operados mediante esa técnica y evaluamos sus resultados clínicos y funcionales.

Resultados La media de rango articular metacarpofalángico fue de $97^{\circ} / 3^{\circ}$, interfalángico proximal de $93^{\circ} / 3^{\circ}$ e interfalángico distal de $82^{\circ} / 1^{\circ}$. El EVA medio fue de 2 y el Quick DASH de 13.

Conclusiones Los resultados obtenidos son comparables con el resto de las técnicas quirúrgicas sin aumento de las complicaciones, lo que nos plantea la posibilidad de comenzar un estudio prospectivo con una sola técnica para tratar la mayoría de los patrones de fractura.

Introduction Although proximal interphalangeal joint fracture dislocations are common in clinical practice, there is no consensus in their management nor specific indications for each kind of fracture. We wondered if it was possible to simplify this algorithm and treat the great majority of this injuries with surgical indication with the Suzuki external fixator in a protocolized way. received

March 9, 2019

accepted

August 21, 2019
DOI https://doi.org/

10.1055/s-0039-1698790. ISSN 1698-8396.
Copyright $\odot 2019$ Thieme Revinter

Publicações Ltda, Rio de Janeiro, Brazil
License terms

(c) (1) $\ominus$ (5) 


\section{Keywords}

- proximal interphalangeal joint

- fracture-dislocation

- dynamic external fixator
Methods We collected 15 patients with fracture patterns type 1 (66.6\%), type 2 (6.6\%), type 3 (20\%) and type 5 (6.6\%) according to Seno classification between 2011 and 2019 who underwent this procedure and they were evaluated about clinical and functional results.

Results The average range of movement in the injured finger was $97^{\circ} /-3^{\circ}$ at the metacarpophalangeal joint, $93^{\circ} / 3^{\circ}$ at the proximal interphalangeal joint and $82^{\circ} / 1^{\circ}$ at the distal interphalangeal joint. The mean VAS value was 2 and the mean Quick DASH score was 13.

Conclusion The results obtained are comparable to the results of other surgical techniques without increasing complications. We consider the possibility of starting a prospective study with this single technique to treat most of these fracture patterns.

\section{Introducción}

La articulación interfalángica proximal tiene un papel crucial en el rango de movimiento de los dedos de la mano, siendo responsable del $85 \%$ del arco de flexión de los mismos. ${ }^{1}$ La fractura-luxación de esa articulación, es muy frecuente en la práctica clínica ${ }^{1-3}$ y surgen distintos patrones de fractura, muchos de ellos de alta complejidad, que se pueden clasificar en relación a la estabilidad, el tamaño y los tipos de fragmento producidos. ${ }^{4,5}$ En relación a esos patrones, varias opciones de tratamiento quirúrgico se han descrito, incluyendo fijación con agujas, reducción abierta y fijación interna, artroplastia con ganchoso o con la placa volar ${ }^{6-11}$ así como un sinfín de sistemas de fijación externa fijos o dinámicos. ${ }^{12-16}$ Todas esas opciones tienen el objetivo de restaurar la estabilidad articular y permitir una movilización precoz para evitar las complicaciones más frecuentes en ese tipo de patologías: la rigidez y el dolor. ${ }^{2,16,17}$ Sin embargo, no existe un consenso ni indicaciones definidas para cada tipo de fractura, lo que supone un importante campo de confusión, sobre todo para el cirujano menos experto que tiene que enfrentarse a lesiones de alta complejidad. ${ }^{18,19}$ Surge la pregunta de si es posible simplificar ese algoritmo y utilizando una sola técnica poder manejar la gran mayoría de las fracturas-luxaciones interfalángicas proximales con indicación quirúrgica. El objetivo de este trabajo es describir los resultados del tratamiento de las fracturas luxaciones de la articulación interfalángica proximal con la utilización del fijador externo dinámico tipo Suzuki.

\section{Material y Métodos}

Se realizó un estudio observacional cuya población incluyó a los pacientes que habían presentado una fractura-luxación de la articulación interfalángica proximal del $2^{\circ}$ al $5^{\circ}$ dedo de la mano y que habían sido intervenidos en Complejo Hospitalario de Toledo, el Hospital Universitario Quirón Madrid y el Hospital Quirón Toledo mediante el fijador externo dinámico tipo Suzuki desde enero de 2011 a enero de 2019. Los criterios de inclusión fueron la presencia de una fractura - luxación de la articulación interfalángica proximal del $2^{\circ}$ al $5^{\circ}$ dedo de la mano, en la que persistía subluxación o ausencia de reducción articular aceptable. La subluxación la definimos como la desviación dorsal o volar de la línea axial diafisaria de la falange media en relación con el centro de la cabeza de la falange proximal y/o el signo de la $\mathrm{V}$ dorsal ${ }^{2,3,5,20}$ y la reducción inaceptable la consideramos cuando existen fragmentos separados por más de $1 \mathrm{~mm}$.

Como criterios de exclusión, se consideró la ausencia de inestabilidad post-reducción de la fractura, la fractura abierta, la infección activa, la lesión neurovascular asociada y la presencia de fisis abierta.

Se recogieron 15 pacientes que cumplían los criterios de inclusión. Entre ellos se encontraron pacientes con patrones de fractura: tipo 1 (66,6\%), tipo 2 (6,6\%), tipo 3 (20\%) y tipo 5 (6,6\%) según la clasificación de Seno y col. ${ }^{4} 4$ pacientes eran mujeres y 11 varones. La edad media fue de 43 años (17-65). En 10 casos el dedo afectado fue el $4^{\circ}$, en 2 casos el $2^{\circ}$ y hubo 2 casos con afectación del $3^{\circ}$ y $5^{\circ}$ dedo. El tiempo medio de seguimiento fue de 23 meses (8-92). El tiempo medio desde el traumatismo hasta la intervención fue de 4,3 días (0-11) (véase - Tabla 1).

Se recogió el tiempo que se mantuvo el fijador, rango articular de las articulaciones metacarpofalángica (MTCF), interfalángica proximal (IFP) e interfalángica distal (IFD) tras la retirada del fijador medido mediante un goniómetro, la funcionalidad definitiva mediante el cuestionario Quick $\mathrm{DASH}^{21}$ y el dolor residual mediante la Escala Visual Analógica (EVA) ${ }^{22}$ recogidos al final del seguimiento (véase -Tabla 2) y las complicaciones asociadas. Se obtuvieron controles radiográficos en el pos-operatorio inmediato y semanalmente en las revisiones hasta la retirada del dispositivo, en los que se evaluó la consolidación y la presencia o ausencia de subluxación de la articulación tras el tratamiento.

\section{Técnica Quirúrgica}

Los pacientes se intervienen mediante una misma técnica, previamente descrita ${ }^{6}$. Antes de comenzar se comprueba bajo escopia la viabilidad de la reducción cerrada de la fracturaluxación mediante tracción y manipulación gentil (-Fig. 1).

Se utilizan tres agujas de kischner $(1,4 \mathrm{~mm}$ la proximal y $1 \mathrm{~mm}$ el resto) introducidas paralelamente bajo control 
Tabla 1 Datos demográficos de la población a estudio

\begin{tabular}{|c|c|c|c|c|c|c|c|c|}
\hline & Sexo & Edad & $\begin{array}{l}\text { Dedo } \\
\text { afecto }\end{array}$ & $\begin{array}{l}\text { Mano } \\
\text { afecta }\end{array}$ & $\begin{array}{l}\text { Clasificación } \\
\text { Seno y } \mathrm{col}^{4}\end{array}$ & $\begin{array}{l}\text { \% superficie } \\
\text { articular }\end{array}$ & $\begin{array}{l}\text { Días hasta } \\
\text { cirugía }\end{array}$ & $\begin{array}{l}\text { Tiempo } \\
\text { seguimiento }\end{array}$ \\
\hline 1 & Mujer & 47 & $5^{\circ}$ dedo & Derecha & Tipo 5 & $>50 \%$ & 2 & 8 \\
\hline 2 & Mujer & 43 & $3^{\circ}$ dedo & Izquierda & Tipo 1 & $30-50 \%$ & 3 & 39 \\
\hline 3 & Hombre & 52 & $2^{\circ}$ dedo & Derecha & Tipo 1 & $30-50 \%$ & 1 & 24 \\
\hline 4 & Hombre & 30 & $4^{\circ}$ dedo & Derecha & Tipo 3 & $30-50 \%$ & 4 & 12 \\
\hline 5 & Hombre & 17 & $2^{\circ}$ dedo & Derecha & Tipo 1 & $<30 \%$ & 1 & 17 \\
\hline 6 & Hombre & 19 & $4^{\circ}$ dedo & Izquierda & Tipo 3 & $>50 \%$ & 7 & 10 \\
\hline 7 & Hombre & 59 & $4^{\circ}$ dedo & Izquierda & Tipo 1 & $<30 \%$ & 11 & 16 \\
\hline 8 & Hombre & 65 & $4^{\circ}$ dedo & Derecha & Tipo 1 & $>50 \%$ & 2 & 13 \\
\hline 9 & Hombre & 46 & $4^{\circ}$ dedo & Izquierda & Tipo 1 & $<30 \%$ & 21 & 17 \\
\hline 10 & Hombre & 62 & $4^{\circ}$ dedo & Izquierda & Tipo 1 & $>50 \%$ & 0 & 92 \\
\hline 11 & Mujer & 28 & $4^{\circ}$ dedo & Derecha & Tipo 1 & $>50 \%$ & 9 & 15 \\
\hline 12 & Hombre & 31 & $2^{\circ}$ dedo & Izquierda & Tipo 3 & $30-50 \%$ & 6 & 18 \\
\hline 13 & Hombre & 51 & $4^{\circ}$ dedo & Derecha & Tipo 1 & $30-50 \%$ & 3 & 20 \\
\hline 14 & Mujer & 39 & $4^{\circ}$ dedo & Derecha & Tipo 2 & $<30 \%$ & 7 & 28 \\
\hline 15 & Hombre & 57 & $4^{\circ}$ dedo & Izquierda & Tipo 1 & $30-50 \%$ & 5 & 20 \\
\hline
\end{tabular}

Tabla 2 Resultados clínicos y funcionales

\begin{tabular}{|c|c|c|c|c|c|c|c|c|c|}
\hline & $\begin{array}{l}\text { Clasificación } \\
\text { Seno y col }\end{array}$ & $\begin{array}{l}\text { Flexión } \\
\text { MTCF }\end{array}$ & $\begin{array}{l}\text { Extensión } \\
\text { MTCF }\end{array}$ & $\begin{array}{l}\text { Flexión } \\
\text { IFP }\end{array}$ & $\begin{array}{l}\text { Extensión } \\
\text { IFP }\end{array}$ & $\begin{array}{l}\text { Flexión } \\
\text { IFD }\end{array}$ & $\begin{array}{l}\text { Extensión } \\
\text { IFD }\end{array}$ & EVA & $\begin{array}{l}\text { Quick } \\
\text { DASH }\end{array}$ \\
\hline 1 & Tipo 5 & 90 & 0 & 120 & 10 & 90 & 0 & 0 & 2,27 \\
\hline 2 & Tipo 1 & 100 & 10 & 50 & -5 & 85 & 0 & 8 & 34,09 \\
\hline 3 & Tipo 1 & 90 & -10 & 85 & 0 & 80 & 0 & 0 & 20,45 \\
\hline 4 & Tipo 3 & 90 & 0 & 90 & 0 & 90 & 0 & 0 & 2,27 \\
\hline 5 & Tipo 1 & 90 & -20 & 100 & 0 & 90 & 0 & 0 & 0 \\
\hline 6 & Tipo 3 & 100 & -10 & 90 & 10 & 90 & 0 & 0 & 9,09 \\
\hline 7 & Tipo 1 & 90 & 0 & 90 & 0 & 50 & 0 & 0 & 0 \\
\hline 8 & Tipo 1 & 120 & -10 & 100 & 5 & 90 & 0 & 5 & 29,54 \\
\hline 9 & Tipo 1 & 90 & 0 & 120 & 0 & 90 & 0 & 0 & 0 \\
\hline 10 & Tipo 1 & 90 & 0 & 80 & 10 & 90 & 0 & 4 & 6,81 \\
\hline 11 & Tipo 1 & 100 & 0 & 90 & 0 & 60 & 10 & 5 & 54,54 \\
\hline 12 & Tipo 3 & 100 & -10 & 90 & 0 & 85 & 0 & 0 & 2,27 \\
\hline 13 & Tipo 1 & 90 & 0 & 100 & 0 & 80 & 0 & 0 & 9,09 \\
\hline 14 & Tipo 2 & 120 & -5 & 90 & 10 & 80 & 0 & 3 & 25 \\
\hline 15 & Tipo 1 & 90 & -5 & 100 & 0 & 85 & 10 & 0 & 4,54 \\
\hline Medias & - & 97 & -3 & 93 & 3 & 82 & 1 & 2 & 13 \\
\hline
\end{tabular}

Abreviaturas: EVA, Escala Visual Analógica; IFD, Interfalángica distal; IFP, Interfalángica proximal; Quick DASH, Incapacidad del Brazo, Hombro y Mano.

radiológico en la cabeza de la falange proximal, base y cabeza de la falange media respectivamente. Cabe destacar la importancia de la colocación de las dos primeras agujas. La primera en el centro de rotación de la cabeza para permitir una movilidad concéntrica articular. La segunda nos permite ajustar la reducción articular, de tal forma que se introduce ligeramente dorsal a la línea axial longitudinal diafisaria lateral si la inestabilidad es dorsal y viceversa si es volar. (-Fig. 2)
Posteriormente, se realiza el doblado de las mismas y se consigue la distracción necesaria con bandas elásticas ( - Fig. 3).

Para conseguir un mejor resultado se debe tener en cuenta una serie de trucos / consejos que resumimos a continuación (-Fig. 3)

- Individualizar el dedo a tratar controlando el resto con cinta adhesiva 


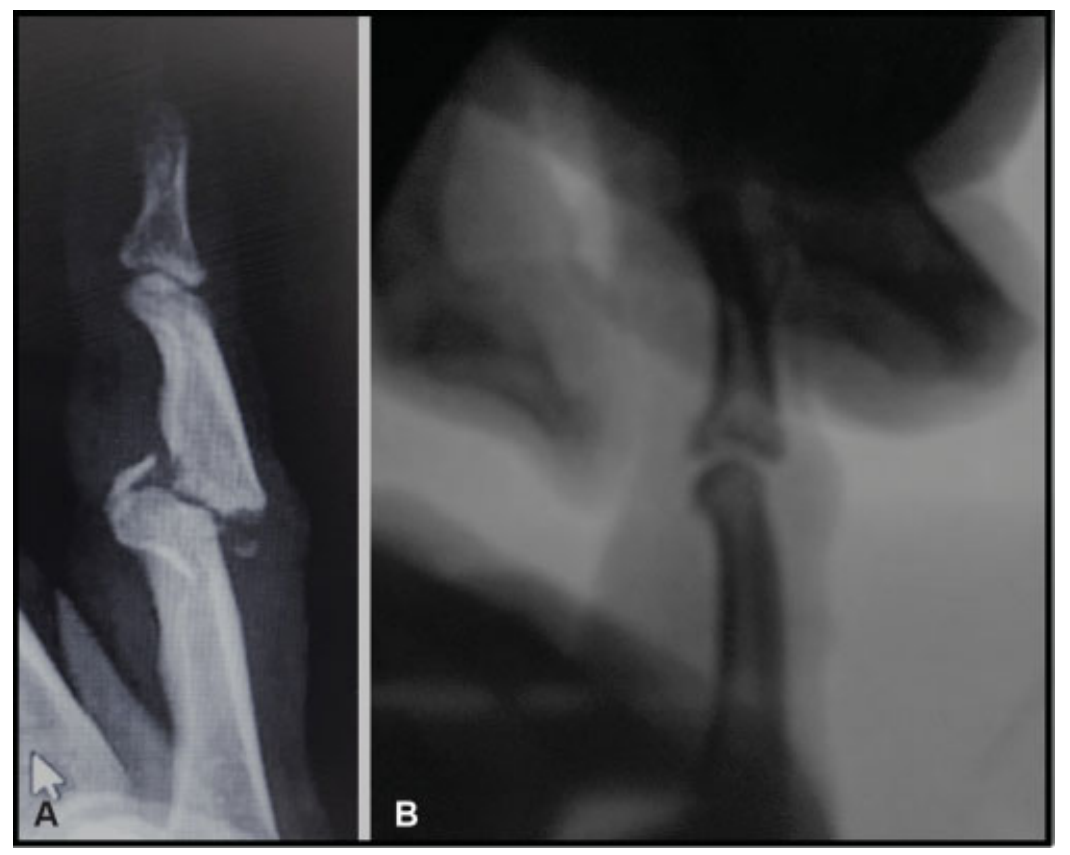

Fig. 1 Comprobación de reducción bajo tracción. a. Fractura luxación de articulación IFP en la que se objetiva subluxación dorsal. b. Reducción de la fractura bajo tracción.

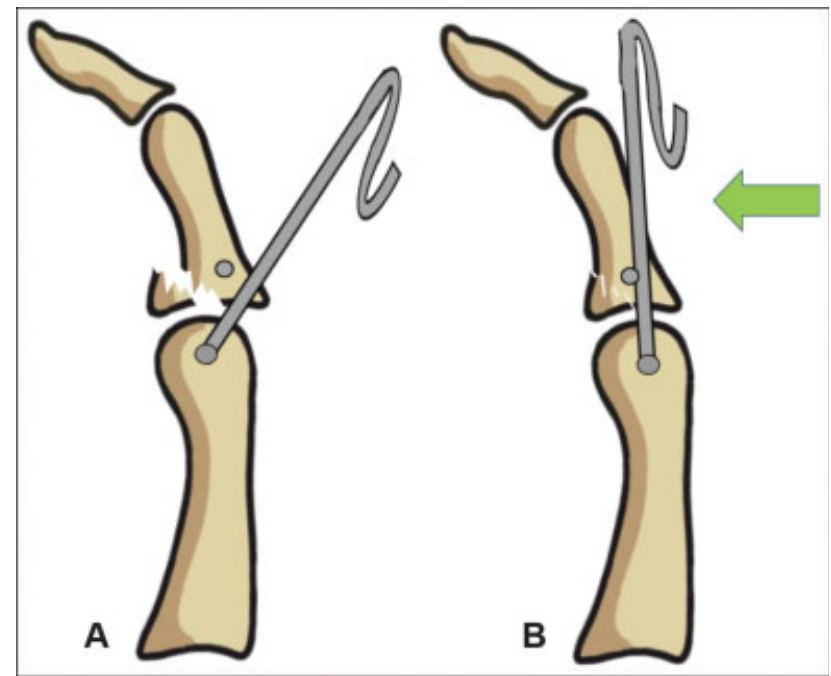

Fig. 2 Esquema de reducción de subluxación dorsal articular. (A) Subluxación dorsal. (B) Reducción de la subluxación dorsal gracias a la aguja intermedia y a la posición dorsal de la primera aguja. Si la subluxación fuese volar la disposición de esas dos agujas sería inversa.

- Realizar la implantación de las agujas de forma ordenada de proximal a distal

- Conseguir proyecciones laterales puras con la escopia.

- Comprobar que todas las agujas se implantan de forma paralela.

- Las gomas elásticas se pueden obtener de la boquilla de cualquier sonda urinaria, utilizando las que necesitemos y comprobando bajo escopia que la tracción ejercida por las mismas es la adecuada.

Se debe comprobar que tras el montaje completo se consigue una reducción completa de la articulación, desapareciendo el signo de la $\mathrm{V}$, y que esa reducción se mantiene en el tiempo una vez retirado el fijador ( - Fig. 4)

\section{Resultados}

El fijador externo se mantuvo una media de 30 días (20-44). El rango articular de flexo extensión medio obtenido fue: $97^{\circ} /-3^{\circ}$ para la articulación MTCF, $93^{\circ} / 3^{\circ}$ para la articulación IFP y $82^{\circ} / 1^{\circ}$ para la articulación IFD. El EVA medio fue de 2 y el Quick DASH de 13 (-Tabla 2).

En el seguimiento se objetivó un mantenimiento de la reducción y una consolidación radiológica completa de la fractura en todos los casos en una media de 37 días.

No observamos ningún caso de infección superficial ni profunda. Observamos un caso de degeneración articular y limitación para la flexión de $50^{\circ}$, pero que no requirió tratamiento posterior.

\section{Discusión}

El tratamiento de la fractura - luxación de la articulación IFP continúa siendo hoy en día, un reto no resuelto, debido, sobre todo, al amplio espectro de patrones de lesiones, que puede requerir desde un tratamiento conservador hasta una cirugía reconstructiva para conseguir un resultado óptimo.

Algunos autores han tratado de establecer un algoritmo terapéutico para su manejo ${ }^{18,20}$ pero el resultado es muy difícilmente reproductible, sobre todo por cirujanos no experimentados en cirugía de la mano.

Los principios de tratamiento descritos por Kiefhaber y Stern $^{5}$ en 1998: restauración de la congruencia articular, movilización precoz y fijación rígida no limitante continúan vigentes, pero en relación a la última premisa existen actualmente estudios que describen las potenciales 


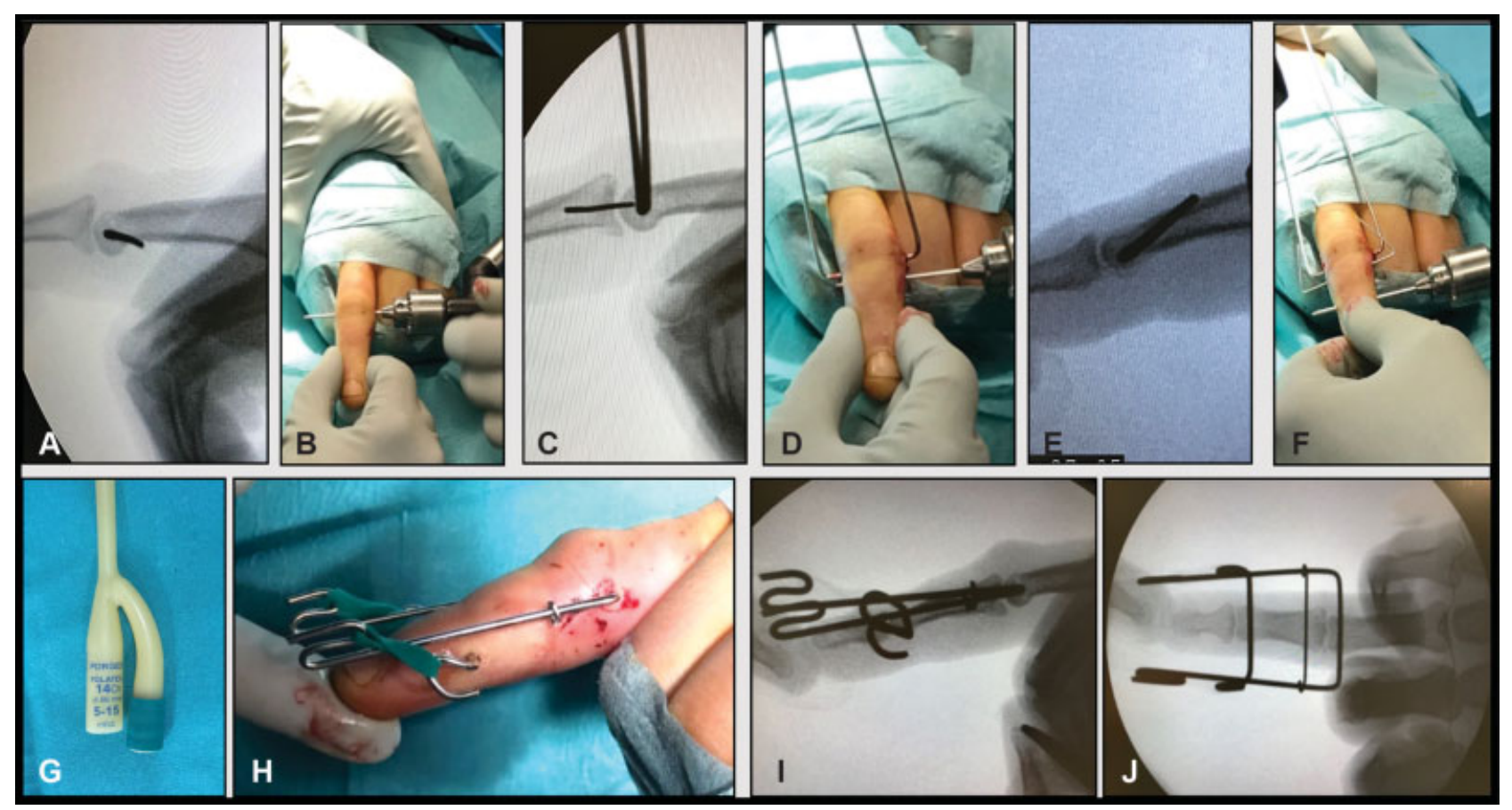

Fig. 3 Montaje del fijador externo tipo Suzuki. (A) Control escópico de la implantación de la primera aguja de Kirshner en el centro de rotación de la cabeza de la falange proximal, paralela a la articulación. (B) Sujeción adhesiva del resto de dedos. (C) Control escópico de la implantación de la segunda aguja en la falange media, distal a la fractura. (D) Doblado a $90^{\circ}$ de los extremos de la aguja proximal e introducción de la aguja media. (E) Control escópico en tercera aguja en el centro de rotación de la cabeza de falange media. (F) Implantación de la tercera aguja. (G) Boquilla de sonda vesical de la que obtenemos las gomas. (H) Doblado de las agujas restantes, ensamblaje del fijador y colocación de las gomas elásticas. (I) Control escópico lateral de la tracción ejercida por las gomas elásticas y comprobación de la reducción de la fractura. (J) Control escópico anteroposterior.

complicaciones de las técnicas quirúrgicas abiertas ${ }^{23}$ como son el dolor, la rigidez ${ }^{2,16,17}$ o la incapacidad de fijación en los casos de fracturas muy conminutas. $7,8,23$

Por ello se considera la opción del fijador externo dinámico como el tratamiento idóneo para ese tipo de lesiones. Desde que el 1986 se describió el primero de esos dispositivos, han ido surgiendo progresivamente diferentes sistemas ${ }^{6,12,13,16,24-26}$ cuya base es la movilización precoz y la restauración de la congruencia articular a través de la reducción de la fractura mediante la ligamentotaxis.,20 Esos muestran buenos resultados en el tratamiento de los diferentes patrones de fractura - luxación, pero las series se centran en un tipo concreto de lesión o no analizan por separado los diferentes patrones en los que se clasifican.

En esta serie se obtiene un rango articular satisfactorio ( $90^{\circ}$ de media), que está en consonancia con lo descrito en la literatura tanto por fijadores externos similares como el de Shen y col., ${ }^{25}\left(83,9^{\circ}\right)$, Finsen y col., ${ }^{27}\left(72^{\circ}\right)$ o Ruland y $\mathrm{col}^{2}{ }^{28}\left(88^{\circ}\right)$ así como por otros fijadores prefabricados como el de Kodama y col $\left(91,2^{\circ}\right)$, MacFarlane y col $\left(85^{\circ}\right)$ y Pélissier y col., $\left(70^{\circ}\right)^{16,24,29}$ y es superior a otras publicaciones en las que se llevó a cabo cirugía abierta, como la de Cheah y col., ${ }^{9}$ $\left(75^{\circ}\right)$.

Respecto a los resultados funcionales, se consiguieron escalas muy satisfactorias, con unas cifras en el Quick DASH bajas (13 de media) similares a lo descrito en la literatura sobre otros sistemas de fijación externa dinámica, ${ }^{17,24,27,29,30}$ a pesar de que como describe Finsen y col., ${ }^{27}$ se comprobó que, en ocasiones, el dispositivo causa una pequeña incomodidad a los pacientes. Además, el dolor residual tras el tratamiento ha resultado mínimo (EVA 2) lo cual ya había sido descrito en estudios previos. ${ }^{12,16,17,27}$

Una ventaja añadida de ese tratamiento es, como describe Shen y col., ${ }^{25}$ la eficacia del mismo en el tratamiento tardío de la fractura luxación de la IFP, logrando en su serie en lesiones con 27.5 días de evolución de media un rango articular medio de $83.9^{\circ}$. En esta serie se cuenta con un paciente con una fractura-luxación de 21 días de evolución cuyo resultado de flexo extensión de la IFP fue de $120^{\circ} / 0^{\circ}$ y sin dolor.

En cuanto a la duración óptima del mantenimiento del fijador, es un punto todavía no completamente definido, existiendo tan solo la recomendación de su mantenimiento de 2 a 7 semanas. ${ }^{17}$ En nuestro caso, se mantuvo una media de 30 días, lo que se sitúa dentro de las recomendaciones y en consonancia con lo realizado en otros estudios ${ }^{24,25,31}$ y por la técnica original. ${ }^{26}$

Como complicaciones cabe destacar un caso que en el que, pese a haber realizado un tratamiento a priori óptimo, presentó rigidez para la flexión de la articulación $\operatorname{IFP}\left(50^{\circ}\right)$. En el estudio radiológico se objetivaron signos indirectos de artrosis de la IFP, complicaciones ya descritas ambas por otros autores..$^{24,27,29} \mathrm{La}$ aparición de rigidez de la articulación IFD, tanto en flexión como extensión, es relativamente frecuente y su etiología no está definida, como ya describe Finsen y col. ${ }^{27}$ Es importante asegurarnos del correcto posicionamiento del fijador y que éste no afecte al aparato extensor ${ }^{27,32}$ y también de conseguir una 


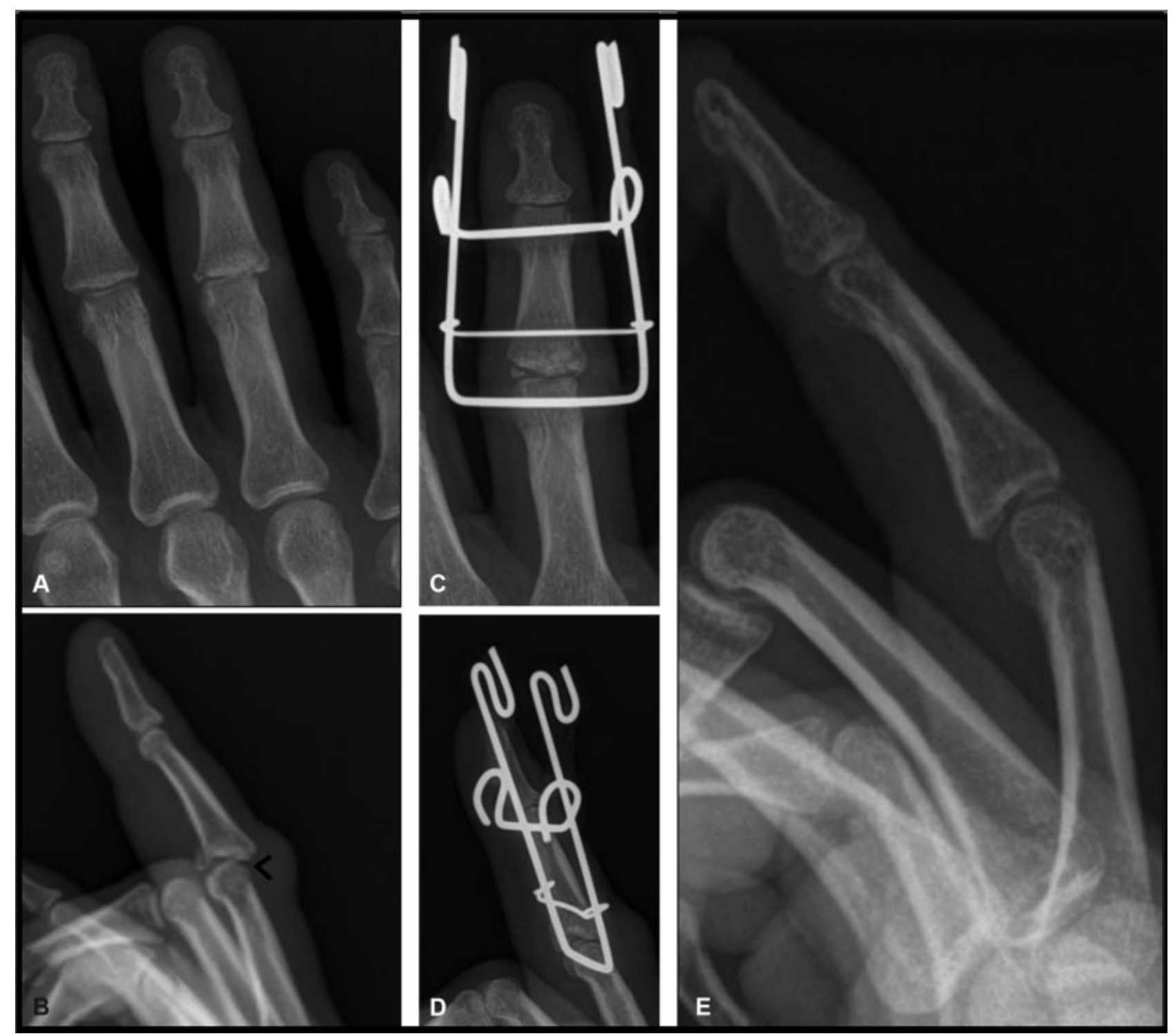

Fig. 4 (A y B) Fractura luxación dorsal. Véase el signo de la $V^{2,3,5,20}$ (C y D) Reducción de la fractura luxación con el fijador externo. (E) Control radiográfico pos-operatorio tras la retirada del dispositivo.

reducción articular correcta, siendo a posteriori el tratamiento rehabilitador el de elección para disminuirla.

Otra de las complicaciones que reporta la literatura es la infección en relación a las agujas, que según las series puede llegar hasta el $28 \%$, pero que generalmente se resuelve con tratamiento de antibiótico y curas locales sin necesidad de retirar el fijador de forma anticipada. ${ }^{17}$ En esta serie, no se produjo ninguna complicación de este tipo.

Resulta difícil realizar una comparación directa de esos resultados en función del tipo de lesión con los estudios previos por heterogeneidad en la clasificación empleada en los mismos por un lado y por otro por nuestra pequeña serie de pacientes recogidos de forma observacional, que no nos permiten sacar resultados significativos.

\section{Conflicto de Intereses}

Los autores del trabajo declaran no tener ningún conflicto de intereses.

\section{Bibliografía}

1 Liodaki E, Xing SG, Mailaender P, Stang F. Management of difficult intra-articular fractures or fracture dislocations of the proximal interphalangeal joint. J Hand Surg Eur Vol 2015;40 (01):16-23

2 Haase SC, Chung KC. Current concepts in treatment of fracturedislocations of the proximal interphalangeal joint. PlastReconstr Surg 2014;134(06):1246-1257

$3 \mathrm{Ng}$ CY, Oliver CW. Fractures of the proximal interphalangeal joints of the fingers. J Bone Joint Surg Br 2009;91(06):705-712

4 Seno N, Hashizume H, Inoue H, Imatani J, Morito Y. Fractures of the base of the middle phalanx of the finger. Classification, management and long-term results. J Bone Joint Surg Br 1997; 79(05):758-763

5 Kiefhaber TR, Stern PJ. Fracture dislocations of the proximal interphalangeal joint. J Hand Surg Am 1998;23(03):368-380

6 Ellis SJ, Cheng R, Prokopis P, et al. Treatment of proximal interphalangeal dorsal fracture-dislocation injuries with dynamic external fixation: a pins and rubber band system. J Hand Surg Am 2007;32(08):1242-1250

7 Burnier M, Awada T, Marin Braun F, Rostoucher P, Ninou M, Erhard L. Treatment of unstable proximal interphalangeal joint fractures 
with hemi-hamate osteochondral autografts. J Hand Surg Eur Vol 2017;42(02):188-193

8 Eaton RG, Malerich MM. Volar plate arthroplasty of the proximal interphalangeal joint: a review of ten years' experience. J Hand Surg Am 1980;5(03):260-268

9 Cheah AEJ, Tan DMK, Chong AKS, Chew WYC. Volar plating for unstable proximal interphalangeal joint dorsal fracturedislocations. J Hand Surg Am 2012;37(01):28-33

10 Ikeda M, Kobayashi Y, Saito I, Ishii T, Shimizu A, Oka Y. Open reduction and internal fixation for dorsal fracture dislocations of the proximal interphalangeal joint using a miniplate. Tech Hand Up Extrem Surg 2011;15(04):219-224

11 Federer AE, Guerrero EM, Dekker TJ, et al. Open Reduction Internal Fixation With Transverse Volar Plating for Unstable Proximal Interphalangeal Fracture-Dislocation: The Seatbelt Procedure. Hand (N Y) 2018:1558944718790063; [Epub ahead of print]

12 Henn CM, Lee SK, Wolfe SW. Dynamic External Fixation for Proximal Interphalangeal Fracture-Dislocations. Oper Tech Orthop 2012;22(03):142-150

13 Damert HG, Altmann S, Kraus A, Infanger M, Sattler D. Treatment of intraarticular middle phalanx fractures using the Ligamentotaxor ${ }^{\circledR}$. Hand (N Y) 2013;8(04):460-463

14 Kapur B, Paniker J, Casaletto J. An alternative technique for external fixation of traumatic intra-articular fractures of proximal and middle phalanx. Tech Hand Up Extrem Surg 2015;19(04): 163-167

15 Sraj S. A Simple Phalangeal External Fixator Using Kirschner Wires and Locking Balls: No Need for Cement or Rubber Bands.J Hand Surg Am 2016;41(07):e217-e221. Doi: 10.1016/j.jhsa.2016.04.017

16 Kodama A, Sunagawa T, Nakashima Y, et al. Joint distraction and early mobilization using a new dynamic external finger fixator for the treatment of fracture-dislocations of the proximal interphalangeal joint. J Orthop Sci 2018;23(06):959-966

17 Lo CH, Nothdurft SH, Park HS, Paul E, Leong J. Distraction ligamentotaxis for complex proximal interphalangeal joint fracture dislocations: a clinical study and the modified pins rubber band traction system revisited. Burns Trauma 2018;6:23

18 Janssen SJ, Molleman J, Guitton TG, Ring D; Science Of Variation Group. What middle phalanx base fracture characteristics are most reliable and useful for surgical decision-making? Clin OrthopRelat Res 2015;473(12):3943-3950

19 Saitta BH, Wolf JM. Treating Proximal Interphalangeal Joint Dislocations. Hand Clin 2018;34(02):139-148
20 Caggiano NM, Harper CM, Rozental TD. Management of Proximal Interphalangeal Joint Fracture Dislocations. Hand Clin 2018;34 (02):149-165

21 http://www.dash.iwh.on.ca/sites/dash/public/translations/Quick DASH_Spanish_Spain_2018.pdf

22 Huskisson EC. Measurement of pain. Lancet 1974;2(7889):1127-1131

23 Stern PJ, Roman RJ, Kiefhaber TR, McDonough JJ. Pilon fractures of the proximal interphalangeal joint. J Hand Surg Am 1991;16(05): 844-850

24 MacFarlane RJ, Gillespie S, Cashin F, Mahmood A, Cheung G, Brown DJ. Treatment of fracture subluxations of the proximal interphalangeal joint using a ligamentotaxis device: a multidisciplinary approach. J Hand Surg Eur Vol 2015;40(08): 825-831

25 Shen XF, Mi JY, Rui YJ, et al. Delayed treatment of unstable proximal interphalangeal joint fracture-dislocations with a dynamic external fixator. Injury 2015;46(10):1938-1944

26 Suzuki Y, Matsunaga T, Sato S, Yokoi T. The pins and rubbers traction system for treatment of comminuted intraarticular fractures and fracture-dislocations in the hand. J Hand Surg [Br] 1994;19(01):98-107

27 Finsen V. Suzuki's pins and rubber traction for fractures of the base of the middle phalanx. J Plast Surg Hand Surg 2010;44(45):209-213

28 Ruland RT, Hogan CJ, Cannon DL, Slade JF. Use of dynamic distraction external fixation for unstable fracture-dislocations of the proximal interphalangeal joint. J Hand Surg Am 2008;33 (01):19-25

29 Pélissier P, Gobel F, Choughri H, Alet JM. Proximal interphalangeal joint fractures treated with a dynamic external fixator: A multicenter and retrospective study of 88 cases. Chir Main 2015;34(05):245-250

30 Colegate-Stone T, Marenah K, Compson J, Tahmassebi R, Tavakkolizadeh A. Functional Outcomes Following Pilon Fractures of the Middle Phalanx Managed with the Ligamentotaxor External Fixator. Hand Surg 2015;20(02):285-289

31 Abou Elatta MM, Assal F, Basheer HM, El Morshidy AF, Elglaind SM, Abdalla MA. The use of dynamic external fixation in the treatment of dorsal fracture subluxations and pilon fractures of finger proximal interphalangeal joints. J Hand Surg Eur Vol 2017;42 (02):182-187

32 Agarwal AK, Karri V, Pickford MA. Avoiding pitfalls of the pins and rubbers traction technique for fractures of the proximal interphalangeal joint. Ann Plast Surg 2007;58(05):489-495 